\title{
Ab initio calculations of magnetic structure and lattice dynamics of Fe/Pt multilayers
}

\author{
P.T. Jochym ${ }^{1, a}$, K. Parlinski ${ }^{1}$, and A.M. Oleś ${ }^{1,2}$ \\ 1 Institute of Nuclear Physics, Polish Academy of Sciences, Radzikowskiego 152, 31342 Kraków, Poland \\ 2 Max-Planck-Institut für Festkörperforschung, Heisenbergstrasse 1, 70569 Stuttgart, Germany
}

Received 4 September 2007 / Received in final form 20 December 2007

Published online 6 February 2008 - (c) EDP Sciences, Società Italiana di Fisica, Springer-Verlag 2008

\begin{abstract}
The magnetization distribution, its energetic characterization by the interlayer coupling constants and lattice dynamics of (001)-oriented Fe/Pt multilayers are investigated using density functional theory combined with the direct method to determine phonon frequencies. It is found that ferromagnetic order between consecutive Fe layers is favoured, with the enhanced magnetic moments at the interface. The bilinear and biquadratic coupling coefficients between Fe layers are shown to saturate fast with increasing thickness of nonmagnetic Pt layers which separate them. The phonon calculations demonstrate a rather strong dependence of partial iron phonon densities of states on the actual position of Fe monolayer in the multilayer structure.
\end{abstract}

PACS. 75.70.Cn Magnetic properties of interfaces (multilayers, superlattices, heterostructures) $-63.22 .+\mathrm{m}$ Phonons or vibrational states in low-dimensional structures and nanoscale materials - 75.50. Bb Fe and its alloys

\section{Introduction}

The technological importance of metallic multilayers consisting of alternating layers of magnetic and nonmagnetic material stimulated both experimental and theoretical studies of these systems which continues over the last two decades. The properties of magnetic multilayers are to a large extent determined by the interface properties. In order to understand whether future applications of multilayers as magnetic data storage materials are possible, their microscopic properties have to be investigated to determine the magnetic interactions on the nanoscale and to understand the dependence of these interactions on the multilayer structure. Since the discovery of composition and annealing dependence of the magnetic properties in Fe/Pt bilayers [1], a number of experimental papers have been devoted to the magnetic properties of the Fe/Pt multilayers $[2,3]$.

The materials themselves are of great technological importance since they are characterized by large uniaxial magnetocrystaline anisotropy energy [4], which in turn leads to high temperature stability of the data magnetically recorded in such a medium [5]. The magnetocrystaline anisotropy energy density has been already analysed $[4,6]$ using ab initio techniques. However, to the best of our knowledge, the dependence of the type of magnetic ordering on the thickness of Fe and Pt layers, as well as

\footnotetext{
${ }^{a}$ e-mail: Pawel.Jochym@ifj.edu.pl
}

lattice dynamics in these systems, have not been investigated with modern computational techniques until now.

The purpose of the present paper is to analyse magnetic ordering and lattice dynamics in the (001)-oriented $\mathrm{Fe} / \mathrm{Pt}$ multilayers using an $a b$ initio approach. We have computed the magnetization distribution in a series of representative multilayer structures with one up to twelve atomic monolayers (MLs) in an iron layer, and one up to five platinum MLs in the spacer layer. The calculations were performed self-consistently and the configurations with ferromagnetic (FM) and antiferromagnetic (AF) order between the consecutive iron layers were obtained and their energies and magnetization distributions were compared with each other. We also report the values of bilinear and biquadratic coupling constants, which quantify the energy difference between the AF and FM configuration of the Fe layers within the model introduced by Fullerton and Bader [7], and depend on the thickness of both magnetic iron layers and (almost) nonmagnetic platinum layers.

The paper is organized as follows. The computational methods are shortly described in Section 2. In Section 3 we present the magnetic properties of $\mathrm{Fe} / \mathrm{Pt}$ multilayers, and systematically investigate the energy difference between the AF and FM configurations, depending on the number of $\mathrm{Fe}$ and $\mathrm{Pt}$ atomic MLs in the considered multilayer structure. The phonon dispersions and the phonon densities of states for two representative Fe/Pt multilayer 


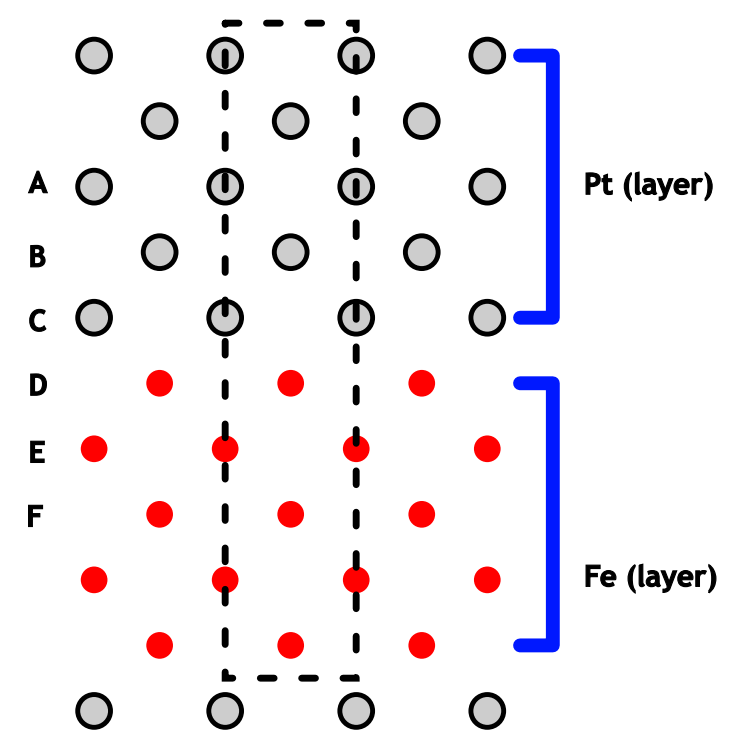

Fig. 1. Side view of a representative $5 / 5 \mathrm{Fe} / \mathrm{Pt}$ multilayer structure before geometry optimization (with five atomic MLs of iron and five atomic MLs of platinum). Non-equivalent MLs in the unit cell are labeled by characters $A-F$; the unit cell in the FM structure, used also for phonon calculations, is indicated by dashed lines (the magnetic unit cell in an AF configuration is twice larger). The bottom row of $\mathrm{Pt}$ atoms is shown only for clarity.

systems are analysed in Section 4. We present a short summary and the main conclusions in Section 5.

\section{Computational methods}

The calculations were carried out with standard density functional theory (DFT) code as implemented in the VASP package [8]. We have used well established ultrasoft generalized gradient approximation (GGA) pseudopotentials [9] with Perdew-Wang 91 gradient correction scheme (as given in Ref. [10]) as input for phonon calculations. The calculations of magnetic structure of the system were carried out with pseudopotentials introduced by Perdew, Burke and Ernzerhof [11,12] (so-called PBE-GGA pseudopotentials) as implemented in the VASP package [9]. The difference in method used for these two calculations does not influence our general conclusions since the results from different methods are not compared with each other. The calculations neglected spin-orbit coupling which may somewhat influence the results, especially for Pt atoms, since they possess fairly strong spin-orbit component.

The series of structures investigated in this paper was derived from the basic (001) bcc iron lattice with platinum atoms replacing iron atoms within platinum layer (by layer we mean one or more MLs of the same material). In this way some MLs of Fe atoms were replaced by $\mathrm{Pt}$ ones. Then the entire multilayer structure was optimized with respect to atomic positions and its unit cell size. The convergence criterion has been set at root mean square (RMS) force $\leq 10^{-7} \mathrm{eV} / \AA$ and RMS stress $\leq 10 \mathrm{MPa}$.

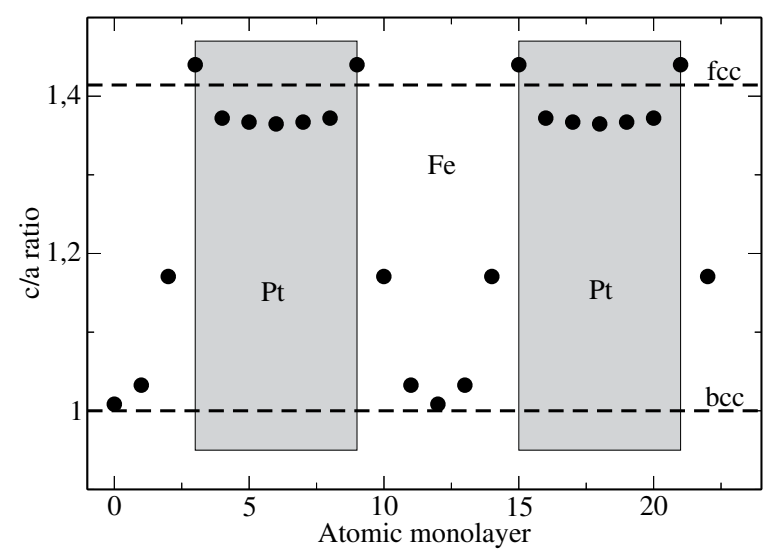

Fig. 2. Inter-layer distance in the $4 / 8$ structure after structure optimization. Dashed lines indicate c/a ratio for ideal bcc (bottom line) and fcc (top line) structures. The shaded areas indicate the extension of the platinum layers.

The atomic forces and magnetic moment density distribution have been calculated using final optimized structures. One example of such a structure before optimization is shown in Figure 1; its unit cell in the FM (ground state) configuration contains atoms belonging to five atomic MLs of iron and to five atomic MLs of platinum, in so-called $5 / 5$ system.

The structure optimization led to substantial changes in the structure of the considered multilayer systems. Namely, the inter-layer distance in the Pt layer increased to the point where this layer adopts the well known experimentally fcc structure. This change is illustrated in Figure 2, showing the local $c / a$ ratio of lattice constants for each atomic monolayer in the structure. It is straightforward to see by analysing these data that the structure of the platinum layer changes from the starting bcc structure (assumed at the beginning of the optimization process) to the fcc lattice - well-known experimentally. This may be interpreted, together with phonon calculations below, as a strong evidence of rather realistic description of the physical properties of the multilayer systems in the presented calculations. The above change starts to show up for quite thin Pt layers - even the system consisting of four Pt atomic MLs shows already the interlayer spacing close to the fcc structure. For thicker Pt layers this effect is very strong (see Fig. 2). The above finding agrees quite well with experimental findings of Antel et al. [13], where a similar process of gradual change from tetragonally distorted bcc (bct) to tetragonally distorted fcc (fct) lattice was observed. The iron layer is quite stable but when it is thin (few atomic MLs) it is prone to be distorted towards the fcc structure. This tendency may be also observed in Figure 2, where the distortion of $c / a$ ratio goes deep into the Fe layer, while almost the whole Pt layer keeps its optimum $c / a$ ratio. Similar structural changes in $\mathrm{Fe} / \mathrm{Pt}$ systems have also been observed experimentally $[2,13]$.

All calculations were carried out for spin-polarized systems with the self-consistent optimization of the magnetization distribution within a given $\mathrm{Fe} / \mathrm{Pt}$ multilayer system. The set of calculated structures contains a range of 
variants from the simplest $1 / 1$ structure (periodic structure with $1 \mathrm{ML}$ of $\mathrm{Fe}$ and $1 \mathrm{ML}$ of $\mathrm{Pt}$ in its unit cell) up to $4 / 8$ (i.e., with 4 atomic MLs of Fe and 8 atomic MLs of $\mathrm{Pt}$ ) in increments of $2 \mathrm{MLs}$, and also, in addition, 1/12 structure (1 ML of Fe and 12 MLs of Pt).

The magnetic properties of $\mathrm{Fe} / \mathrm{Pt}$ multilayers were investigated systematically (see Sect. 3 ) by changing the thickness of Fe and Pt layers in a periodic system consisting of four layers, i.e., twice larger than that shown in Figure 1, with two Fe and two Pt spacer layers interchanged with each other, with periodic boundary conditions. Several different starting configurations for the magnetization distribution were used in all cases in order to obtain unbiased results for a given system. Here we have used a similar approach to that employed to investigate $\mathrm{Fe} / \mathrm{FeSi}$ multilayers in our recent paper [14].

Starting from equilibrium multilayer configurations obtained as a result of electronic structure calculations, we investigated lattice dynamics for two representative Fe/Pt multilayers: $4 / 4$ and 5/5 systems (see Fig. 1 ), reported in Section 4. Thereby we used the so-called direct method of lattice dynamics calculation [15]. It follows the early idea of calculating the force constants with ab initio methods [16] by implementing the full crystal symmetry given by the crystallographic space group [15]. As such, it is able to achieve high accuracy and became a very powerful computational tool in the last decade. By construction it allows one to determine accurate phonon frequencies at the high symmetry points in the reciprocal space, and gives otherwise the phonon spectra with high accuracy, if the force constants decrease fast enough with increasing distance, as in the present case.

The formulation of reference [15] provides an algorithm to calculate force constants automatically for a system of any size. The procedure employed in the present study used displacements of $0.03 \AA$ in each non-equivalent direction for every non-equivalent atom. The number of necessary displacements was thus determined by the system symmetry and by the size of the multilayer unit cell. Next, the matrices of force constants were found using the singular value decomposition method. Finally, the dynamical matrix was constructed and diagonalized for each wave vector $\mathbf{k}$.

\section{Magnetic properties}

When Fe layers are separated by layers of nonmagnetic Pt spacer in a $\mathrm{Fe} / \mathrm{Pt}$ multilayer, the first question concerns the distribution of magnetization over the Fe atoms. In the case of platinum spacer we have found that the interface proximity substantially enhances magnetic moments of iron atoms. This can be clearly observed for the $4 / 4$ $\mathrm{Fe} / \mathrm{Pt}$ structure, with magnetic moments $m \sim 2.8 \mu_{B}$ and $\sim 2.5 \mu_{B}$ for the Fe atoms at the interface and in the inner Fe ML (Fig. 3), respectively, and with differences less than $2 \%$ between the FM and AF configuration.

Also in larger systems we have found that the magnetic moments $m$ at the interface are systematically enhanced and are rather close to $3.0 \mu_{B}$, i.e., the $3 d$ electrons are

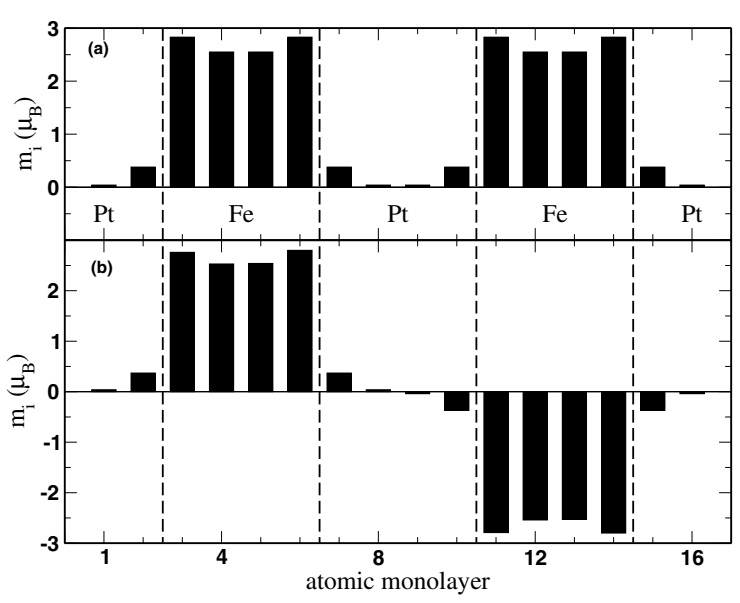

Fig. 3. Distribution of magnetic moments $m_{i}$ in the $4 / 4$ multilayer structure consisting of two layers with four Fe MLs separated by $\mathrm{Pt}$ spacer with four Pt MLs, as obtained for two magnetic configurations: (a) two magnetic unit cells in the FM ground state, and (b) magnetic unit cell of the (locally stable) AF order between two neighbouring Fe layers.

almost fully polarized. In contrast, the moments at the $\mathrm{Fe}$ atoms of inner MLs are smaller and gradually approach the bulk value, $m_{\mathrm{Fe}} \simeq 2.2 \mu_{B}$, with increasing thickness of the Fe layer. While the later behavior may be expected and in fact is observed experimentally [13], the magnetization increase at the interface $\mathrm{Fe}$ atoms characterized by a lower coordination of $\mathrm{Fe}$ atoms is not obvious. We interpret it as a manifestation of electron correlation effects which increase local moments at Fe atoms when the kinetic energy of $3 d$ electrons is decreased by a lower Fe coordination [17]. In agreement with this interpretation, the moment enhancement at the interface becomes even more pronounced in the extreme case of a single iron ML, where $\mathrm{Fe}$ atoms have only platinum coordination and their magnetic moments are increased up to the level of saturation $m \simeq 3.0 \mu_{B}$, i.e., by $35 \%$ over the bulk iron value $m_{\mathrm{Fe}} \simeq 2.2 \mu_{B}$ (the magnetic moments of Pt atoms at the interface are here quite small $m_{\mathrm{Pt}} \simeq 0.3 \mu_{B}$ ).

The above magnetic moment amplification effect at the interface in case of $\mathrm{Pt}$ spacer is remarkably different from the behaviour found recently for $\mathrm{Fe} / \mathrm{FeSi}$ multilayers [14]. There, the magnetic polarization at interface Fe atoms is reduced, while the magnetic moments at $\mathrm{Fe}$ atoms of the second ML are somewhat enhanced. This demonstrates, that the moment enhancement may occur only in case of a metallic spacer, while we suggest that the electronic states at an insulating interface behave more like molecular bonding and thus $3 d$ electrons are partly bound and the magnetization is reduced.

In addition, large magnetic moments at $\mathrm{Fe}$ atoms of the metallic interface induce weak FM polarization in the neighbouring Pt ML $\left(m \simeq 0.4 \mu_{B}\right.$ in case of $4 / 4$ structure of Fig. 3). The induced magnetization of the Pt layer has been observed experimentally [13] to be on the same level as calculated here. There is however a difference in the distribution of the magnetic moment inside Pt layer - the present results indicate large enhancement at the 
interface, while the experimental results suggest more uniform distribution of the moment across the whole Pt layer. This difference may be connected with the contribution of spin-orbit interaction which, according to the experimental results [13], is absent or less pronounced in the case of iron-transition metal multilayers. It might be also to certain extent attributed to interdiffusion in the measured sample, following the suggestion of reference [13]. Unfortunately, such effects cannot be included in the electronic structure calculations at present.

Our main goal in investigating the magnetic properties of multilayers is to establish the dependence of the favoured magnetic ordering in the underlying system on the thickness of the spacer (here $\mathrm{Pt}$ ) layer and to characterize it by certain global parameters. Here we follow a phenomenological model of Fullerton and Bader [7] introduced to describe the energy difference between an AF and a FM configuration of a given multilayer structure. In this model it is assumed that the energy difference between an AF and a FM configuration of the system, may be expressed by the following simple formula:

$$
\Delta E=E_{\mathrm{AF}}-E_{\mathrm{FM}},
$$

$$
E_{\mathrm{AF}(\mathrm{FM})}=J_{1} \mathbf{m}_{1} \cdot \mathbf{m}_{2}+J_{2}\left(\mathbf{m}_{1} \cdot \mathbf{m}_{2}\right)^{2}+E_{0}+E_{m} .
$$

Here $J_{1}$ and $J_{2}$ are the bilinear and biquadratic coupling parameters, respectively, while $\mathbf{m}_{1}$ and $\mathbf{m}_{2}$ are magnetic moments of two interacting layers normalized per area unit (only the interactions between the nearest neighbour magnetic layers are considered). $E_{0}$ and $E_{m}$ are reference energies of nonmagnetic structure and magnetic energy of the isolated layers, respectively. Note that the magnetic moments depend on the number of Fe MLs, and are practically the same for the AF and the FM configuration of a given multilayer structure (see below).

The derivation of the parameters $\left\{J_{1}, J_{2}\right\}$ in equation (2) requires the calculation of energies of the (locally stable) AF, FM and nonmagnetic variants of each investigated structure separately. Here we use the same method as the one introduced recently for Fe/FeSi multilayers [14]. Note that the same result could also be obtained by calculations performed for two systems with spins oriented in two different directions (e.g. the reference system and the one rotated by 90 degrees), but this would require employing the non-collinear spins technique which is more involved and more technically demanding than the approach used here. In each case we determined these locally stable configurations and next analysed the results in order to extract information about the interlayer exchange couplings.

Furthermore, the procedure used here requires the values of magnetic moments $\mathbf{m}_{l}$ of two coupled layers $l=1,2$. The magnetic moments have been derived from the calculated spin-polarized electronic structure by numerical integration of the magnetic moment density in the spherical volume with radius $r=1.3 \AA$ around each atom. These individual moments were subsequently summed up within respective layers. We have verified that the resulting moments for each layer were practically the same (the

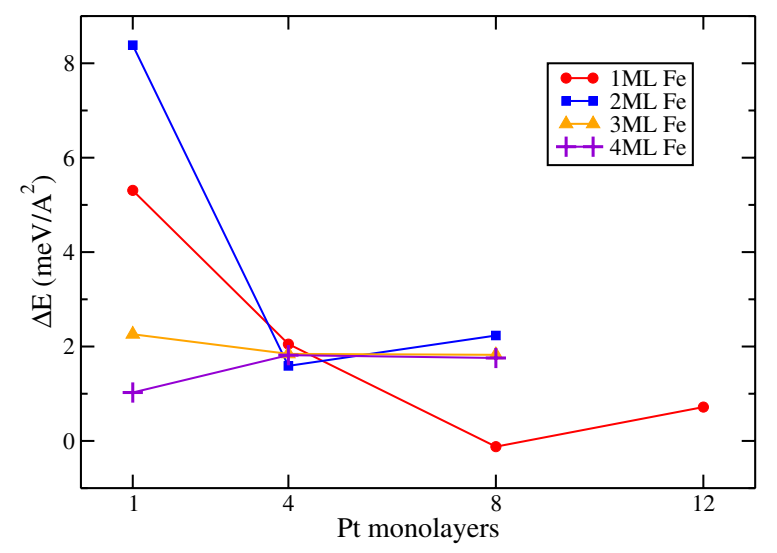

Fig. 4. Energy difference $\Delta E$ (1) between AF and FM order of Fe layers in $\mathrm{Fe} / \mathrm{Pt}$ multilayers for $1-4$ atomic Fe MLs, as a function of increasing thickness of the Pt spacer layer.

difference is less than $\leq 1 \%$ ) for both AF and FM configuration of the same structure. Further details concerning this procedure of calculating the coupling parameters $\left\{J_{1}, J_{2}\right\}$ were described in the previous paper [14].

The energy difference $\Delta E(1)$ is presented in Figure 4 as a function of the increasing thickness of platinum spacer layer for several $\mathrm{Fe} / \mathrm{Pt}$ multilayers. The data shows a clear preference towards the FM ordering of consecutive Fe layers already for the systems with only up to four Fe MLs. Note, however, that the energy difference $\Delta E$ decreases (with the exception of a single Fe ML) with increasing thickness of the iron layer, while the platinum layer consists of a single ML.

The above behaviour is again different from the $\mathrm{Fe} / \mathrm{FeSi}$ system, where the order between the Fe layers is AF for thin layers, and becomes FM only for sufficiently thick FeSi spacer. Furthermore, it is quite remarkable that the energy difference $\Delta E$ for all the considered Fe/Pt multilayers except for the system with one Fe ML converges quite fast to a constant value with the increasing thickness of the Pt layer. This feature appears to be again universal with exception of the system with a single iron ML which does not contain bulk-like iron atoms with only iron coordination, and thus is expected to behave in a different way.

The thickness dependence of the coupling coefficients between Fe layers $J_{1}$ and $J_{2}$, see equation (2), are presented in Figure 5. It is interesting to compare them with the ones obtained before for metal-insulator Fe/FeSi multilayers. There are some similarities and some differences between the characters of their thickness dependence in $\mathrm{Fe} / \mathrm{FeSi}$ multilayers [14], and in the present metallic Fe/Pt ones. First, in both cases, one finds rather weak dependence of $J_{2}$ on the thickness of the spacer layer (top right panel of Fig. 5). In case of one or two Fe MLs there is also no noticeable convergence of $J_{2}$ with increasing thickness of the spacer layer, while for thicker Fe layers the values of $J_{2}$ are very small indeed for $8 \mathrm{Pt}$ MLs and suggest that this parameter becomes gradually irrelevant for increasing thickness of spacer layers. Second, there is a qualitative difference in $J_{2}$ behaviour as a function of iron layer 


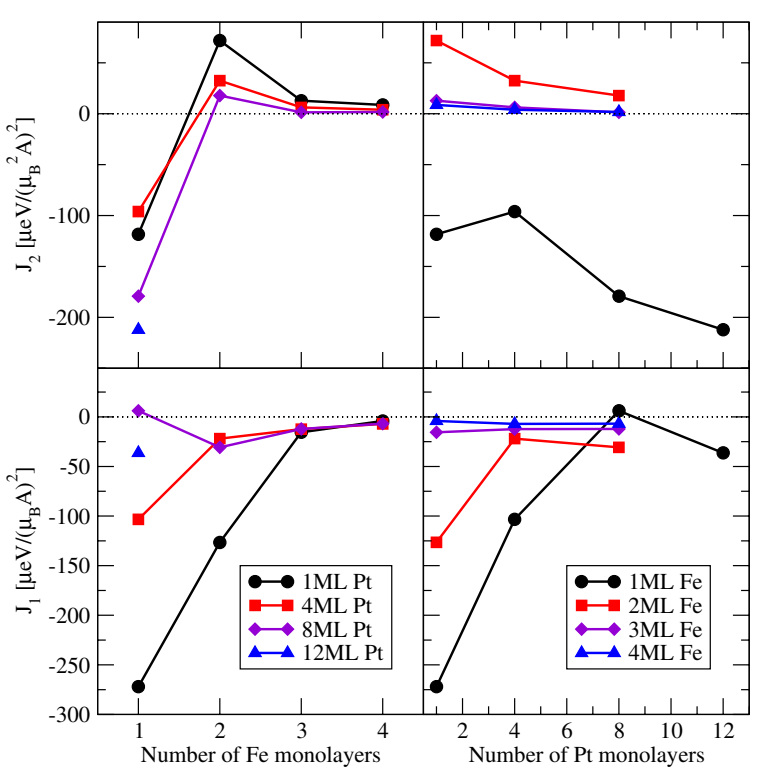

Fig. 5. Bilinear $J_{1}$ (bottom) and biquadratic $J_{2}$ (top) coupling coefficients defined by equation (2) as obtained for Fe/Pt multilayers for increasing thickness of iron layer (left) and platinum spacer layer (right).

thickness with the one found in Fe/FeSi systems. In the case of $\mathrm{Fe} / \mathrm{FeSi}$ multilayers one finds consistent and close to the exponential decay of $J_{2}$, while for $\mathrm{Fe} / \mathrm{Pt}$ we could spot some kind of oscillatory shape in the $J_{2}$ plot (top left panel of Fig. 5). This we interpret as a manifestation of the metallic behaviour, in contrast to the gradual decay of the coupling parameter $J_{2}$ in an insulating medium.

On the contrary, the behaviour of the bilinear coupling parameter $J_{1}$ (bottom part of Fig. 5 ) shows more similarities between $\mathrm{Fe} / \mathrm{FeSi}$ and $\mathrm{Fe} / \mathrm{Pt}$ case. In both systems the values of the $J_{1}$ converge quite fast with increasing thickness of both iron and spacer layer. In case of the iron-thickness dependence (bottom left panel of Fig. 5) the asymptotic value appears to be close to zero, while the data suggest that it is finite for the spacer-thickness dependence (bottom right panel of Fig. 5).

A single ML of iron is again remarkably different from other multilayer systems with thicker Fe layers. The values of $J_{2}$ coupling constant are in this case negative and rather large, in contrast to thicker Fe layer. Furthermore, the values of $\left|J_{2}\right|$ do not decrease with increasing thickness of $\mathrm{Pt}$ spacer, unlike for thicker layers. Also the values of $J_{1}$ show a different behaviour from that found for ticker layers, and the change to positive $J_{1}$ found at $8 \mathrm{Pt}$ MLs followed by a large negative value for $12 \mathrm{Pt}$ MLs is even somewhat surprising. It suggests that the magnetization density within Pt layers exhibits oscillations, which in some cases may even lead to weak AF coupling (see also Fig. 4). Altogether, all these features confirm that the behaviour of a single Fe ML is dominated by the interface, and follows from a rather strong FM polarization of $\mathrm{Pt}$ atoms at the metallic interface, which extends into the Pt layers.

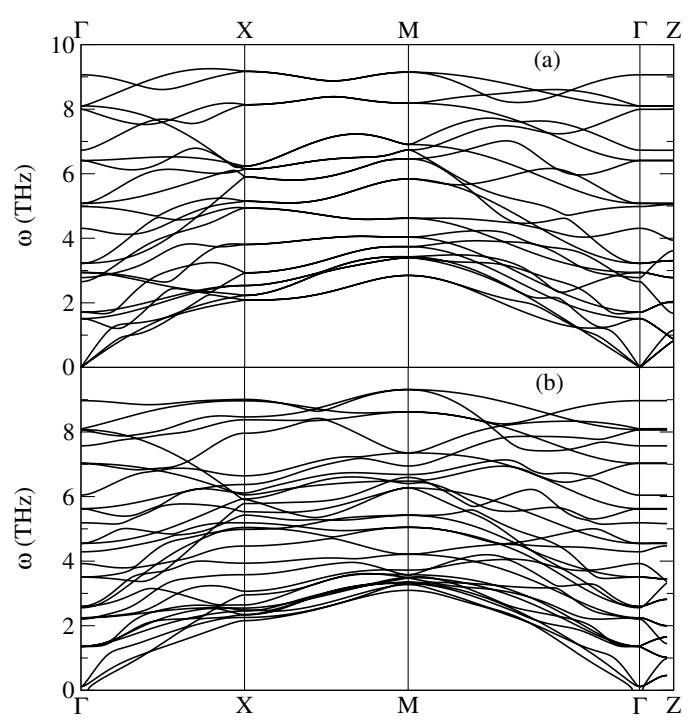

Fig. 6. Phonon dispersion relations of the Fe/Pt multilayer structures in FM configuration as functions of wave vector $\mathbf{k}$ along high symmetry directions in the Brillouin zone for: (a) 4/4 multilayer, and (b) 5/5 multilayer of Fig. 1 . The high symmetry points in units of $\frac{2 \pi}{a}$ are: $\Gamma=(0,0,0), X=(1,0,0)$, $M=(1,1,0), Z=(0,0,1)$.

\section{Lattice dynamics}

We complete the discussion of the physical properties of $\mathrm{Fe} / \mathrm{Pt}$ multilayers by presenting the phonon spectra for selected optimized structures. In order to reach unbiased conclusions, the lattice dynamics has been calculated for two systems, using the $2 \sqrt{2} \times 2 \sqrt{2} \times 1$ supercell for the $4 / 4$ multilayer structure, and the $\sqrt{2} \times \sqrt{2} \times 1$ supercell for the $5 / 5$ one, respectively (the side view of the $5 / 5$ structure is shown in Fig. 1). These calculations are rather demanding and do not allow us to treat more atoms in a unit cell at present, but the unit cells of these two systems are already large enough to identify certain generic features of the phonon spectra in the investigated family of $\mathrm{Fe} / \mathrm{Pt}$ multilayers. To understand them better, we first summarize shortly the results of the electronic structure calculations - they yield a tetragonal structure with $a=b=4.0246 \AA$ and $c=13.7906 \AA$ lattice constants. The former value is only slightly larger than iron lattice parameters (we found $a=4.0188 \AA$ for a test calculation with the same setup). This means that there are only small stresses in the multilayer - judging from the compressibility of the iron structure not higher than of the order of $0.3 \mathrm{GPa}$. The interlayer spacing results are the same as described above in Section 2.

The equilibrium configuration was determined by comparing the total energies of several possible stackings of $\mathrm{Pt}$ layer on Fe layer. The structure of the layers themselves was chosen following the experimental evidence that the bcc iron and fcc Pt are present in this material. The crystal structure of the $4 / 4$ system is given by the $P 4 / \mathrm{nmm}$ space group, labelled as 129 (group $D_{4 h}^{7}$ in Schoenflies notation), and for the $5 / 5$ one it is $P 4 m m, 99\left(C_{4 v}^{1}\right)$ respectively. We have verified that the structure with the lowest energy 


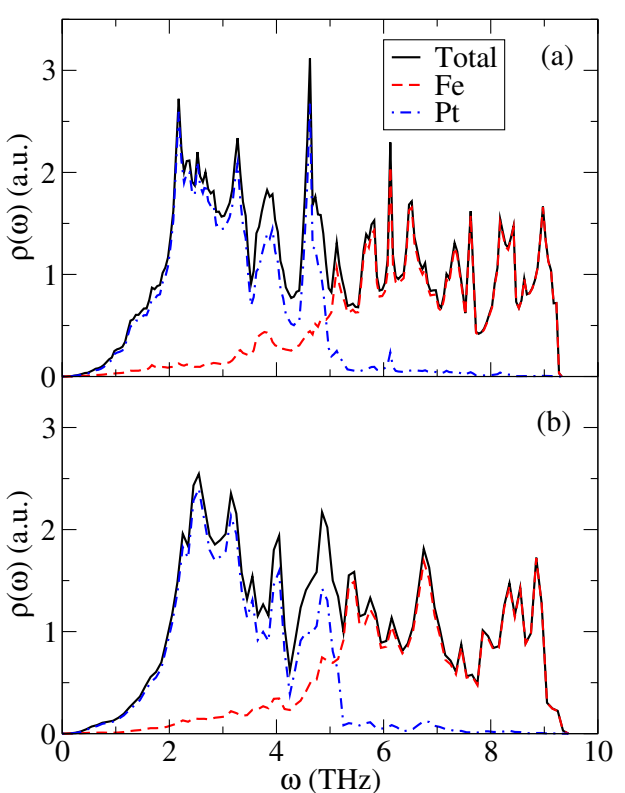

Fig. 7. Total phonon density of states $\rho(\omega)$ (solid lines) and partial phonon densities of states for $\mathrm{Fe}$ (dashed lines, red) and $\mathrm{Pt}$ (dashed-dotted, blue) atoms, respectively, as obtained for the FM ground state with: (a) 4/4 multilayer, and (b) 5/5 multilayer, represented schematically in Figure 1.

is the one with a straightforward continuation of lattice points from bcc lattice of iron to fcc lattice of platinum, as shown in Figure 1 and described in Section 2.

The calculated phonon dispersion relations for the $4 / 4$ and $5 / 5$ structures are shown in Figure 6 . It is clear that the shape of the dispersion curves is quite similar, and the energy range of phonon spectra is the same for both systems. The main difference is in the number of branches, due to the different symmetries of the investigated multilayers. Thus one finds 3 acoustic and 27 (21) optic phonon branches for the $5 / 5(4 / 4)$ multilayer, and some differences in the detailed dispersions of individual phonon modes. However, in Figure 7 we can clearly see that these differences do not affect significantly the distribution of spectral weight in the total phonon density of states $\rho(\omega)$, and the overall frequency ranges for the individual contributions to $\rho(\omega)$. This observation gives further support to the present choice of the supercell - even the $\sqrt{2} \times \sqrt{2} \times 1$ supercell used for the $5 / 5$ system is large enough to obtain meaningful and representative results for the phonon density of states, while for detailed analysis of the dispersion curves it is necessary to consider instead a larger $2 \sqrt{2} \times 2 \sqrt{2} \times 1$ supercell, as in the $4 / 4$ system.

The phonon density of states function $\rho(\omega)$ in Figure 7 consists of two distinct contributions - heavier platinum atoms contribute with lower frequencies, while the vibrations of iron atoms are seen in the upper part of $\rho(\omega)$, well separated from the lower part. The structure of the upper band of the phonon density of states $\rho(\omega)$ is clearly influenced by the contributions of the bulk-like vibrations of the inner iron layers, while the wide background is added by the interface atomic layers.

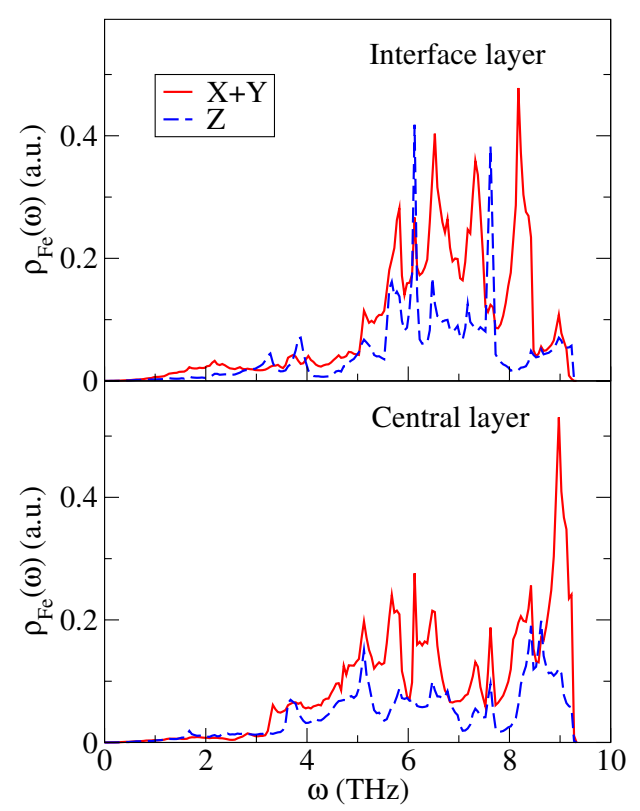

Fig. 8. Partial phonon densities of states $\rho_{\mathrm{Fe}}(\omega)$ projected at $\mathrm{Fe}$ atoms of the $4 / 4 \mathrm{Fe} / \mathrm{Pt}$ multilayer structure in FM configuration for: interface (top), and central (bottom) Fe ML. In each panel the densities of states for $X-Y$ and $Z$ polarizations are plotted separately by solid (red) and dashed (blue) lines, respectively.

While an experimental verification of the theory concerning the phonon dispersions in the $\mathrm{Fe} / \mathrm{Pt}$ multilayers (Fig. 6) is not possible at the moment, we suggest that the projected phonon densities of states at iron atoms $\rho_{\mathrm{Fe}}(\omega)$ could be measured by the nuclear inelastic scattering, which make use of the Mössbauer effect [18]. Since the Mössbauer experimental techniques are sensitive only to iron atoms and depend on polarization for the considered type of layered structure, we extracted the projected phonon densities of states for two different iron MLs in the $4 / 4$ structure, with $X-Y$ planar vibrations, and with perpendicular $Z$ vibrations, see Figure 8 . As the projected phonon density of states for non-equivalent inner iron MLs of the $5 / 5$ system are rather similar to each other, we analyse in detail $\rho_{\mathrm{Fe}}(\omega)$ obtained for the $4 / 4$ system, as it shows already the general trends representative of the considered structures.

The differences between the $X-Y$ and $Z$ phonon polarizations in Figure 8 are remarkable. While the spectral weight for the $Z$ polarization is more uniformly distributed, one finds characteristic maxima in the $X-Y$ density of states near $\omega \simeq 6.5 \mathrm{THz}$ and $\omega \simeq 8.8 \mathrm{THz}$ for the central layer (bottom of Fig. 8). At the interface (top of Fig. 8) an additional structure at $\omega \simeq 7.2 \mathrm{THz}$ was identified, while the maximum at high energy is moved down to $\omega \simeq 8.0 \mathrm{THz}$, with low spectral weight above $8.3 \mathrm{THz}$. Also for the $Z$ polarization the spectral intensity in the high energy part $\omega>8 \mathrm{THz}$ is reduced.

Altogether, the phonon spectra show the quasi-twodimensional character of the investigated multilayer structure, with large spectral weight in the range of $5.5<\omega<$ 8.5 $\mathrm{THz}$ for the interface, and a broader and extending 
up to $\sim 9.3 \mathrm{THz}$ projected phonon density of states $\rho_{\mathrm{Fe}}(\omega)$ for the inner Fe ML (Fig. 8). This feature follows from the coupling to heavier Pt atoms at the interface, so the phonon frequencies are there reduced. The opposite situation was found for Fe/FeSi interface [14], where the spectral weight was shifted to higher frequency range for the modes which involve light Si atoms at the interface. However, the projected phonon density of states found for the inner $\mathrm{Fe} \mathrm{ML}$ in $\mathrm{Fe} / \mathrm{Pt}$ system has several features (overall distribution of spectral weight, the positions of maxima) which make it similar to that of the inner Fe ML in $\mathrm{Fe} / \mathrm{FeSi}$ multilayer. This confirms that the lattice vibrations depend predominantly on the local coordination of particular Fe atoms, which follows from the short-range character of interatomic forces.

\section{Summary and conclusions}

We have calculated the magnetic properties and lattice dynamics of the set of $\mathrm{Fe} / \mathrm{Pt}$ multilayer systems of varying thickness. In order to demonstrate the universal stability of the FM configuration between neighbouring Fe layers, we investigated spacer thickness dependence of the energy difference between the AF and FM structures. This energy difference was quantified using the bilinear $\left(J_{1}\right)$ and biquadratic $\left(J_{2}\right)$ coupling coefficients. It is quite remarkable that the values of $J_{1}$ and $J_{2}$ saturate fast with increasing thickness of Pt layer, indicating that the FM order of consecutive Fe layers is hardly influenced by the $\mathrm{Pt}$ spacer. These data may be therefore considered to be representative of larger systems as well.

A systematic analysis of the magnetic structures shows that the magnetic properties of $\mathrm{Fe} / \mathrm{Pt}$ multilayer are quite different from those of $\mathrm{Fe} / \mathrm{FeSi}$ multilayers, where a crossover from the AF to FM interlayer coupling (between Fe layers) was found instead, with increasing thickness of the FeSi spacer layer [14]. In the case of Pt spacer the tendency towards FM order is well pronounced, and the magnetic moments of Fe atoms at the interface are enhanced. This agrees with earlier experimental findings which revealed rather robust tendency towards FM order in the $\mathrm{Fe} / \mathrm{Pt}$ multilayers [3].

The phonon study has demonstrated that the phonon spectra consist of several modes, reflecting the geometry of a considered system with large unit cell. Platinum and iron vibrations are rather well separated from each other, and the vibrations of iron atoms contribute predominantly at higher energies $\omega>3.5 \mathrm{THz}(\omega>5 \mathrm{THz}$ in case of the interface layer), as shown by the respective partial phonon densities of states. The phonon spectra projected on iron atoms depend rather strongly on the multilayer geometry, and differ distinctly from those of the bulk Fe [19], both for the interface and for the inner Fe MLs, at least when the iron layer consists of not more than five atomic MLs, as considered in the present study. We believe that the maxima in the partial phonon density of states projected on iron atoms shown in Fig. 8 should be fairly easy to observe and verify experimentally. Therefore, we hope that the present theoretical predictions concerning lattice dy- namics will help to understand the intricate dependence of the phonon spectra on the multilayer structure.

Summarizing, the Fe/Pt multilayers exhibit not only interesting magnetic properties, but have also non-trivial and rich lattice dynamics, characterized by rather strong dependence of the partial phonon intensities on the position of an Fe monolayer in the structure. It is believed that the presented results are representative for the $\mathrm{Fe} / \mathrm{Pt}$ systems and will stimulate future experiments.

This work was partially supported by the European Community under FP6 contract No. NMP4-CT-2003-001516 (DYNASYNC). A.M. Oleś acknowledges support by the Foundation for Polish Science (FNP).

\section{References}

1. M. Kitada, Thin Solid Films 146, 21 (1987)

2. M. Sakurai, Phys. Rev. B 50, 3761 (1994); T. Koide, T. Shidara, K. Yamaguchi, A. Fujimori, H. Fukutani, N. Nakajima, T. Sugimoto, T. Katayama, Y. Suzuki, Phys. Rev. B 53, 8219 (1996); R. Bertacco, F. Ciccacci, Phys. Rev. B 57, 96 (1998); M. Abid, H. Lassri, R. Krishnan, M. Nývlt, Š. Višnovský, J. Magn. Magn. Mat. 214, 99 (2000)

3. A. Simopoulos, E. Devlin, A. Kostikas, A. Jankowski, M. Croft, T. Tsakalakos, Phys. Rev. B 54, 9931 (1996)

4. A. Sakuma, J. Phys. Soc. Jpn 63, 3053 (1994)

5. A. Moser, K. Takano, D.T. Margulies, M. Albrecht, Y. Sonobe, Y. Ikeda, S. Sun, E.E. Fullerton, J. Phys. D: Appl. Phys. 35, R157 (2002)

6. G.H.O. Daalderop, P.J. Kelly, M.F.H. Schuurmans, Phys. Rev. B 44, 12054 (1991)

7. E.E. Fullerton, S.D. Bader, Phys. Rev. B 53, 5112 (1996)

8. G. Kresse, J. Furthmüller, Comput. Mater. Sci. 6, 15 (1996)

9. G. Kresse, D. Joubert, Phys. Rev. B 59, 1758 (1999)

10. J.P. Perdew, J.A. Chevary, S.H. Vosko, K.A. Jackson, M.R. Pederson, D.J. Singh, C. Fiolhais, Phys. Rev. B 46, 6671 (1992)

11. J.P. Perdew, K. Burke, M. Ernzerhof, Phys. Rev. Lett. 77, 3865 (1996)

12. P.E. Blöchl, Phys. Rev. B 50, 17953 (1994)

13. W.J. Antel, M.M. Schwickert, Tao Lin, W.L. O'Brien, G.R. Harp, Phys. Rev. B 60, 12933 (1999)

14. P. Jochym, K. Parlinski, A.M. Oleś, Phys. Rev. B 73, 224411 (2006)

15. K. Parlinski, Z.Q. Li, Y. Kawazoe, Phys. Rev. Lett. 78, 4063 (1997); K. Parlinski, software PHOnON, Cracow (2004)

16. K. Kunc, R.M. Martin, Phys. Rev. Lett. 48, 406 (1982); M.T. Yin, M.L. Cohen, Phys. Rev. B 26, 3259 (1982); W. Frank, C. Elsässer, W. Fähnle, Phys. Rev. Lett. 74, 1791 (1995); B.B. Karki, S.J. Clark, M.C. Warren, H.C. Hsueh, G.J. Ackland, J. Crain, J. Phys.: Condens. Matter 9, 375 (1997); G.J. Ackland, M.C. Warren, S.J. Clark, J. Phys.: Condens. Matter 9, 7861 (1997)

17. A.M. Oleś, G. Stollhoff, Phys. Rev. B 29, 314 (1984); G. Stollhoff, A.M. Oleś, V. Heine, Phys. Rev. B 41, 7028 (1990); Phys. Rev. Lett. 76, 855 (1996)

18. R. Rohlsberger, J. Phys.: Condens. Matter 13, 7659 (2001)

19. J. Łażewski, P. Piekarz, A.M. Oleś, K. Parlinski, Phys. Rev. B 74, 174304 (2006) 